\title{
Research on the Fitting Methods of Calibration Curve for Atomic Emission Spectrum Analysis
}

\author{
Ribo Ning ${ }^{1, a}$, Qian $\mathrm{Li}^{1, \mathrm{~b}}$, Songning $X \mathrm{u}^{1, \mathrm{c}}$ \\ ${ }^{1}$ School of Science, Shenyang Ligong University, Shenyang, Liaoning, 110159, China \\ aemail: ningribo@163.com, bemail: liqian198331@163.com, cemail: xsn_201309@126.com
}

Keywords: Spectrum analysis; Calibration curve; Nonlinear fitting; Weighted formula

\begin{abstract}
Based on the Lomakin-Schiebe formula and by means of nonlinear weighted maximum likelihood estimate (NWMLE), the calibration curve fitting methods for atomic emission spectrum (AES) analysis were strictly deduced and the regression equations on the curve parameters were obtained. All the parameters of the calibration curve by numerical calculation methods were calculated. The certified reference materials (CRM) of solid copper alloy were excited by the high-speed spark spectrum excitation source; the spectral data were measured by the photoelectric direct reading spectrometer. The experimental laws on the relationship with relative standard deviation (RSD) of the measured data and the element mass concentration and the new form empirical formula for the weighted were worked out. The calibration curves of two elements in the bronze alloy CRM were established in line with the experimental data and the NWMLE method, and the instrument constants and self-absorption coefficients were measured. The results showed that: $334.5 \mathrm{~nm}$ emission line of $\mathrm{Zn}$ in range of $0.20 \sim 0.96 \%$ concentrations was self-absorption, the 349.3nm emission line of $\mathrm{Ni}$ in range of $0.10 \sim 3.96 \%$ concentrations was clearly self-absorption. Analysis of the experimental data indicated the NWMLE method was of feasibility and broad applicability on the AES analysis.
\end{abstract}

\section{Introduction}

In spectral analysis work, to establish the calibration curve is an important basic work, the accuracy of a calibration curve established determines the accuracy of the results, any deviation from the calibration curve and the result will be reflected in the analysis results in the form of systematic errors. Processing spectral data is now mostly done by calculation software, due to limitations of the software features, the operator cannot use methods other than software functionality calibration curve .The majority of commercial software use a linear fitting method [1] in establishing calibration curve at direct reading for AES analysis, Such as using a polynomial or piecewise polynomial approximation to fit. These methods are easily approximated using a polynomial fitting method less than 3 times [2], instead of the strictly quantitative AES analysis or the actual volatility of detection signal. Although there was domestic literature which reported [3] that, changes in the slope of calibration curve and the situation that the correlation coefficient becomes smaller using a linear weighting method applied by instrument manufacturers, however there wasn't instructions of specific weighting methods or causes of those conclusions. The constant which obtained by polynomial approximation fitting method doesn't have any physical meaning, cannot be used at the measuring course of self-absorption coefficient of the spectrum or the instrument constant. Therefore, this paper provide a strictly nonlinear weighted maximum likelihood estimation method for calibration curve parameters, according to the AES quantitative theory and mathematical statistics theory, and thus directly self-absorption coefficient and the instrument constant can be obtained.

\section{Fitting Methods}

\section{Linear Fitting Methods.}

AES quantitative analysis is based on that there is a function relationship between elements 
concentration to be analyzed and emission lines intensity. This relationship is very complex, generally have a nonlinear relationship [4]: In certain experimental conditions, element concentration within a certain range, element emission line intensity $I$ and element concentration $C$ meet Lomakin-Schiebe formula:

$$
I=a C^{b}
$$

Where: $a$ is instrument constant about evaporation and stimulated emission, $b$ is constant related to self-absorption.

In the formula (1), when $I$ is relative intensity and $C$ is concentration ratio, the relationship described are applicable to the concentration of a larger range. Generally the method used to fit calibration curve is not equation (1) which estimate the value of $a$ and $b$ directly, but polynomial (2) approximately:

$$
C=K_{0}+K_{1} I+K_{2} I^{2}+K_{3} I^{3}+\ldots \ldots
$$

According to experience, in general formula (2) up to take the first four, there is a certain degree of subjectivity in the choose of the order. Although higher order polynomial is much closer to the experimental points than lower order in general, but occasional deviation which actually exist may be masked and lead to the occurrence of systematic deviation. The same problems also exist when we use lower order polynomial fitting curve.

In the mathematical model adopted in Polynomial fitting, changes in analysis precision instrument with concentration are generally ignored, So that a greater degree of deviation from the calibration curve at the low point of the concentration, bring systematic errors in the analyzed results in the low range of the concentration. Although in the polynomial fitting method, artificial "weighting method" can be used to compensate for this shortcoming, but this cannot change the approximation of polynomial fitting method fundamentally.

For fitting equation (1), the simplest way is to be converted into a linear form [5], shown at equation (3):

$$
Y=\ln I=\ln a+b \ln C
$$

Do the linear fit according to the relationship $Y \sim \ln C$, thereby we can easily obtain the values of $a$ and $b$. Although this approach is very simple, but it also did not consider the prerequisite for the establishment in theory: $Y(\ln I)$ obeys normal distribution. From mathematical statistics theory, this prerequisite is equivalent to: the measured value $I$ obeys lognormal distribution. Practical experience and statistical theory indicate: under normal circumstances the measured values obey normal distribution instead of lognormal distribution.

There is significant disparity between normal distribution and lognormal distribution, it increased with the increase degree of deviation of lognormal distribution. The deviation of the results obtained by the two distributions increase, with the increase of the relative standard deviation of measured values; it also increases significantly with the change of experimental system conditions. Therefore, in this article we use nonlinear weighted maximum likelihood estimation method to do calibration curve fitting in accordance with the measured value $I$ is in normal distribution, thereby obtain the best estimates of the parameters $a$ and $b$.

\section{Nonlinear Fitting Methods.}

Using equation (1) to do calibration curve fitting, is classified non-linear fitting, theoretical derivation of this method is more cumbersome, and the regression equation to the parameter is non-linear, usually cannot be solved by algebraically analytical method, only can obtain the best estimate of the parameters using the numerical solution at a certain accuracy. For simplicity on the mathematical model, this article does not involve the presence of inter- element effects and spectral interference, also ignore the effects of spectral background and photodiode dark current, only discuss the relationship between net signal of the elements emission line intensities and its concentrations.

The same CRM was measured several times on element emission line intensities under repeatability conditions, the measured value $I$ is a random variable. By mathematical statistical theory and practical experience it shows that, generally the random variable $I$ obey normally 
distribution [6], and it's the combined effects of a number of causal factors, denoted as:

$$
I \sim N\left(\mu, \sigma^{2}\right)
$$

Where, $\mu$ is the mathematical expectation of $I, \sigma^{2}$ is the variance of $I$.

In the absence of systematic errors $\mu$ is the real value, can be given by equation (1), at this time there are:

$$
\mu=a C^{b}
$$

By the formula (4), (5) there is:

$$
I \sim N\left(a C^{b}, \sigma^{2}\right)
$$

Standard normal form is of the formula (6), as equation (7):

$$
I / \sigma \sim N\left(\frac{a C^{b}}{\sigma}, 1\right)
$$

Suppose that the numbers of CRM provided in the experiment is $n$, concentration value of an element in the CRM is, respectively: $C_{1}, C_{2}, \ldots \ldots, C_{\mathrm{n}}$, measured values corresponding to the intensity of emission lines are $: I_{1}, I_{2}, \ldots \ldots, I_{\mathrm{n}}$, standard deviation corresponding with $I$ are: $\sigma_{1}$, $\sigma_{2}, \ldots \ldots, \sigma_{\mathrm{n}}$. Using experimental points $\left(C_{\mathrm{i}}, I_{\mathrm{i}}\right)$ (or by the point $\left.\left(C_{\mathrm{i}}, I_{\mathrm{i}} / \sigma_{\mathrm{i}}\right)\right)(i=1,2, \ldots \ldots, n)$ to estimate parameters $a$ and $b$ of theoretical curve, is normal non-linear regression problem. In this paper, we use the maximum likelihood estimation method, likelihood function of sample $I_{\mathrm{i}} / \sigma_{\mathrm{i}}$ is:

$$
\begin{aligned}
& L_{n}=\prod_{i=1}^{n} \frac{1}{\sqrt{2 \pi}} e^{-\frac{1}{2}\left(I_{i} / \sigma_{i}-a C_{i}^{b} / \sigma_{i}\right)^{2}}=\frac{1}{(\sqrt{2 \pi})^{n}} e^{-\frac{1}{2} Q(a, b)} \\
& Q(a, b)=\sum_{i=1}^{n} \frac{1}{\sigma_{i}^{2}}\left(I_{i}-a C_{i}^{b}\right)^{2}
\end{aligned}
$$

Standard deviation $\sigma_{\mathrm{i}}$ of random variables $I_{\mathrm{i}}$ can be estimate under certain confidence level from the experimental standard deviation $S_{\mathrm{i}}$, using $1 / S_{\mathrm{i}}{ }^{2}$ as the weighting value. In the production of a calibration curve, to give statistical estimate of the variance $\sigma_{\mathrm{i}}^{2}$ of intensity values of emission line in the CRM of all the elements one by one is troublesome, weights can be determined based on empirical data, relationship between $\sigma$ and $C$ (or $\sigma$ and $I$ ) can be summed from experimental data.

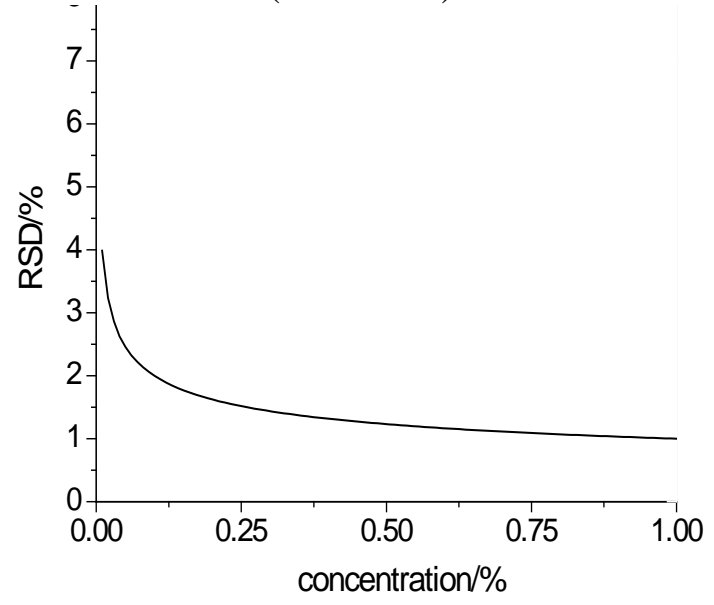

Fig. 1 Curve of RSD and concentration

By summarizing the spark excitation experimental data of various elements of standard solid copper alloy samples found that: there are inversely proportional to the law between the analysis results RSD and concentration, I.e., the lower the concentration is and the larger the analysis result RSD is, the higher the concentration is and the smaller the analysis results RSD is, speed of RSD reduced gradually slows down with the concentration increase, until the RSD tends to a constant. Law mentioned above can be expressed by the image of the "L-type" curve in Fig.1, and the curve in Fig. 1 can be described by the empirical formula (10): 


$$
R S D(C \%)=R S D(1 \%) \times \frac{1}{C^{K}}
$$

Where $\operatorname{RSD}(C \%)$ is the relative standard deviation of the analysis result when the concentration is $C \%$; Where RSD (1\%) is the relative standard deviation of $1 \%$ of the concentration of the analysis result when the concentration is $1 \%$, most of the elements of $\operatorname{RSD}(1 \%)$ is at 1 to $2 \%$ using spark discharge light source and photoelectric direct reading spectrometer, where $K$ is about 0.3 .

P. W. J. M. Boumans et al depicted the "L-type" of calibration curve with complex formula in the form of roots. It should be noted that, the rule in reference [7] is derived from the experimental data by ICP source and in liquid sample, and result in this paper is derived from the experimental data by electric discharge source and in solid CRM. The Underlying causes of the consistent resulting of two different excitation light source need to be considered.

Formula (10) is applied to a larger concentration in the range: $0.00 \mathrm{x} \sim \mathrm{xx} . \mathrm{xx} \%$. RSD under the range of $0.10 \%$ concentration changes little, it can be considered constant within the range or a narrow range of concentration. If the element concentration of the CRM to be analyzed fills the above conditions, there is the following relationship:

$$
R S D=\frac{\sigma}{C}=\sigma_{0} \text { 即 } \sigma=\sigma_{0} C
$$

Wherein, $\sigma_{0}$ is a constant, its value related to the element. This equation shows that: standard deviation to the measured value $I$ and element concentration is proportional, in a certain range. Formula (11) into equation (9), there is:

$$
Q(a, b)=\frac{1}{\sigma_{0}^{2}} \sum_{i=1}^{n} \frac{1}{C_{i}^{2}}\left(I_{i}-a C_{i}^{b}\right)^{2}
$$

Through mathematical statistical theory it shows that: when likelihood function $L_{\mathrm{n}}$ takes a maximum value, corresponding value to parameter is the best estimate value [8]. $Q$ corresponding takes a minimum value at this time. At the minimum, the following two partial derivatives are zero:

$$
\begin{aligned}
& \frac{\partial Q(a, b)}{\partial a}=-2 \sum_{i=1}^{n}\left(I_{i}-a C_{i}^{b}\right) C_{i}^{b-2}=0 \\
& \frac{\partial Q(a, b)}{\partial b}=-2 a \sum_{i=1}^{n}\left(I_{i}-a C_{i}^{b}\right) C_{i}^{b-2} \ln C_{i}=0
\end{aligned}
$$

Represented by the formula (13), (14), there is the regression equation on the parameter b:

$$
B(b)=\sum_{i=1}^{n} I_{i} C_{i}^{b-2} \sum_{i=1}^{n} C_{i}^{2 b-2} \ln C_{i}-\sum_{i=1}^{n} I_{i} C_{i}^{b-2} \ln C_{i} \sum_{i=1}^{n} C_{i}^{2 b-2}=0
$$

Equation (15) cannot be solved by analytical methods, numerical analysis solution can be used, in certain accuracy the value of $b$ can be obtained, then the value of $a$ can be obtained by the following formula:

$$
a=A(b)=\frac{\sum_{i=1}^{n} I_{i} C_{i}^{b-2}}{\sum_{i=1}^{n} C_{i}^{2 b-2}}
$$

\section{Application Results and Discussions}

\section{Application Results.}

In this experiential, we use the US Baird photoelectric direct reading spectrometer, $\mathrm{KH}-5$ spark excitation, unidirectional discharge, silver auxiliary electrode of $\Phi 5 \mathrm{~mm}$, cone apex of $90^{\circ}$, analysis gap 5mm, pre-excitation 6 seconds/416 volts, exposure 8 seconds/208 volts, 99.996\% purity argon, pressure at $0.17 \mathrm{MPa}$, flow of $9.43 \mathrm{l} / \mathrm{min}$, and $\mathrm{Zn}$ wavelength of $334.5 \mathrm{~nm}$, Ni wavelength of 349.3nm, experimental sample is copper alloy CRM. 
Using NWMLE methods, the experimental data in the alloy of two elements [9] is processed, calibration curve is obtained, shown in Fig. 2 and Fig. 3, and is comparing with the results with polynomial fit (polyfit), shown in Table 1 and Table 2. The best estimate value of the parameters $a$ and $b$ of the calibration curve is obtained directly.

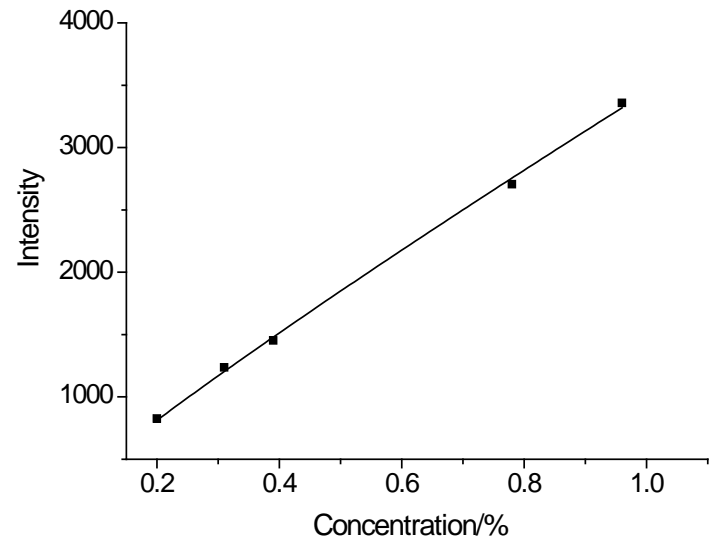

Fig. 2 Calibration curve of Zinc

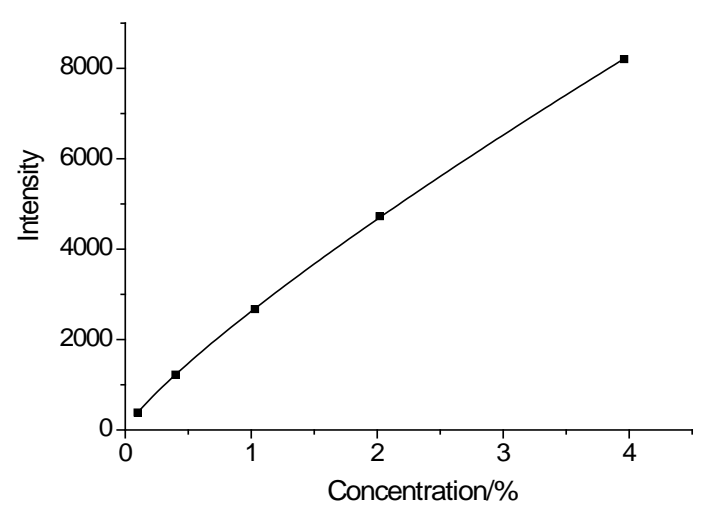

Fig.3 Calibration curve of Nickel

Table 1 Contrast analysis results of Zinc

\begin{tabular}{ccccccc}
\hline $\begin{array}{c}\text { CRM } \\
\text { Serial number }\end{array}$ & Intensity & Concentration /\% & $\begin{array}{c}\text { Polyfit } \\
\text { result/\% }\end{array}$ & $\begin{array}{c}\text { Polyfit } \\
\text { deviation }\end{array}$ & $\begin{array}{c}\text { Method } \\
\text { result/\% }\end{array}$ & $\begin{array}{c}\text { Method } \\
\text { deviation }\end{array}$ \\
\hline $1 \#$ & 827 & 0.20 & 0.196 & $+1.9 \%$ & 0.200 & $-0.17 \%$ \\
$2 \#$ & 1237 & 0.31 & 0.321 & $-3.5 \%$ & 0.316 & $-2.0 \%$ \\
$3 \#$ & 1453 & 0.39 & 0.387 & $+0.85 \%$ & 0.380 & $+2.7 \%$ \\
$4 \#$ & 2705 & 0.78 & 0.767 & $+1.6 \%$ & 0.768 & $+1.6 \%$ \\
$5 \#$ & 3357 & 0.96 & 0.966 & $-0.59 \%$ & 0.981 & $-2.1 \%$ \\
\hline
\end{tabular}

Table 2 Contrast analysis results of Nickel

\begin{tabular}{ccccccc}
\hline $\begin{array}{c}\text { CRM } \\
\text { Serial number }\end{array}$ & Intensity & Concentration /\% & $\begin{array}{c}\text { Polyfit } \\
\text { result/\% }\end{array}$ & $\begin{array}{c}\text { Polyfit } \\
\text { deviation }\end{array}$ & $\begin{array}{c}\text { Method } \\
\text { result/\% }\end{array}$ & $\begin{array}{c}\text { Method } \\
\text { deviation }\end{array}$ \\
\hline $1 \#$ & 385 & 0.10 & 0.096 & $+4.4 \%$ & 0.100 & $+0.04 \%$ \\
$2 \#$ & 1223 & 0.40 & 0.410 & $-2.4 \%$ & 0.401 & $-0.21 \%$ \\
$3 \#$ & 2670 & 1.03 & 1.022 & $+0.75 \%$ & 1.024 & $+0.56 \%$ \\
$4 \#$ & 4727 & 2.02 & 2.022 & $-0.13 \%$ & 2.035 & $-0.72 \%$ \\
$5 \#$ & 8204 & 3.96 & 3.960 & $+0.01 \%$ & 3.946 & $+0.35 \%$ \\
\hline
\end{tabular}

\section{Discussion.}

The results in the table shows that: deviation of the CRM 5\# is ten thousandth, obviously too small, deviation of the CRM $1 \#$ and $2 \#$ is from $1.9 \sim 4.4 \%$, the difference compared with the minimum of $0.01 \%$ is up to 190 to 440 times. From the empirical data curve in graph1, it shows that: when the ratio between the highest and the lowest concentration is 39.6 times, its ratio of the RSD is about 10 times, obviously result of polynomial method is inconsistent with the experimental data. In addition, parameters in polynomial curve fitting method has no physical meaning, cannot provide such guidance message for experimental method as instrument constant, self-absorption spectroscopy and so on.

Correct analysis result can be obtained based on the standard deviation of measured values at different concentration using the method, the obtained parameters have a physical meaning: self-absorption coefficient of Zn $b=0.882219$, self-absorption coefficient of Ni element $b=$ 0.832263 . It can be seen: under these experimental conditions, $334.5 \mathrm{~nm}$ emission line of $\mathrm{Zn}$ element has the presence of self-absorption phenomenon in the concentration of $0.20 \sim 0.96 \%, 349.3 \mathrm{~nm}$ emission line of $\mathrm{Ni}$ element has an apparent self-absorption phenomena in the concentration of 0.10 3.96\%; physical meaning of instrument constant a is the spectral intensity values of emission lines of an element at 1\% concentration, it can be obtained that intensity of the spectral line of $\mathrm{Zn}$ at 
$334.5 \mathrm{~nm}$ with the concentration of $1 \%$ is $a=3415.8$; intensity of the spectral line of $\mathrm{Ni}$ at $349.3 \mathrm{~nm}$ with the concentration of $1 \%$ is $a=2617.3$.

When using numerical methods for solving equation (15), we need to assign initial value for $b$, under normal circumstances, $0<b \leq 1$, although under special experimental conditions $b>1$, but $b$ is not too huge. Numerical solution of $b$ can be obtained by setting the initial value of $b$ to 1 .

When the concentration of the analyzed elements and matrix elements in the CRM varies little, formula (11) can be used to get weighted. When the range of analyzed elements concentration is high, for example: ICP-OES concentration dynamic range is up to 4-5 orders of magnitude, the correlation coefficient is close to 1.0000 in three orders of magnitude [10], then the formula (11) is no longer applicable, the standard deviation of the measured value $I$ can be derived from formula (10) to replace formula (11), as a weighted formula for high range of concentration, the instrument constant and self-absorption coefficient can be obtained according to NWMLE methods herein, the regression equation form of new parameters is more complex.

\section{Conclusion}

Using the Lomakin-Schiebe formula nonlinear weighted fitting methods, weighted based on the reciprocal of the squared experimental standard deviations of spectral signal measured value, equations of curve parameters were obtained from the maximum likelihood estimation method, curve parameters were derived by numerical methods, established an accurate calibration curve, and instrument constant and self-absorption coefficients could be measured by the curve. NWMLE method can overcome the subjectivity in the choice of the order of polynomial times when using polynomial calibration curve. In AES analysis, the curve of the relationship between the concentration and the relative standard deviation of the analyzed results in spark excitation and ICP-OES are both showed "L-type", it can be used as a weighted basis to do calibration curve fitting.

\section{Acknowledgement}

In this paper, the research was sponsored by China National Natural Science Fund (Project No. 61378042).

\section{References}

[1] K.Danzer. Analytical Chemistry. Theoretical and Metrological Fundamentals[M]. Berlin: Springe, 2007. 130 134.

[2] A. A. Boitsov. Journal of Applied Spectroscopy[J], 1989, 51(5):1191.

[3] ZHANG Zhuo-yong, PIAO Zhe-xiu, ZENG Xian-jin. Chinese Journal of Applied Chemistry[J], 1991, 8(6): 10.

[4] JIANG Zu-cheng, CHEN Xin-kun. Modern Atomic Emission Spectrum Analysis[M]. Beijing:

Science Press, 1999. 161.

[5] Peter C. Meier. Richard E. Zund. Statistical Methods in Analytical Chemistry. 2nd ed[M]. New York: 2000. 129.

[6] CHEN Rong-jiang, ZHANG Wan-qin. The Theory of Probability and Statistics[M]. Beijing: Peking University Press, 2006. 122.

[7] P.W.J.M. Boumans, Fresenius Z. Anal. Chem[J]. 1979, 299: 345.

[8] S. Weisberg. Applied Linear Regression 2nd ed. Translated by WANG Jing-long, LIANG Xiao-jun, LI Bao-hui[M]. Beijing: China Statistics Press. 1998. 13 14.

[9] LIANG Yun-ling. Chinese Journal of Spectroscopy Laboratory[J], 1998, 10(3): 3-159.

[10] Norman N. Sesi, Timothy K. Starn, and Gary M.Hieftje. Fresenius' Journal of Analytical Chemistry[J]. 1993, 346(6-9): 545-549. 